\title{
Equivariant Giambelli formula for the symplectic Grassmannians - Pfaffian Sum Formula
}

\author{
Takeshi Ikeda $\|^{\prime}$ and Tomoo Matsumura $\|^{\text {: }}$ \\ ${ }^{1}$ Okayama University of Science, Okayama, Japan \\ ${ }^{2}$ KAIST, Daejeon, Korea
}

\begin{abstract}
We prove an explicit closed formula, written as a sum of Pfaffians, which describes each equivariant Schubert class for the Grassmannian of isotropic subspaces in a symplectic vector space.

Résumé. On démontre une formule close explicite, écrite comme une somme de Pfaffiens, qui décrit toute classe de Shubert équivariante pour la Grassmannienne des sous-espaces isotropes dans un espace vectoriel symplectique.
\end{abstract}

Keywords: symplectic Grassmannian, Schubert calculus, Giambelli formula, Pfaffian, signed permutation, $k$-strict partition

\section{Introduction}

The classical Giambelli formula expresses a general Schubert class of the Grassmannian as the determinant of a matrix whose entries are the so-called special Schubert classes. A special Schubert class is defined by the locus of subspaces having excess intersection with a fixed linear subspace. These classes also coincide with the Chern classes of the universal quotient bundle over the Grassmannian. Various extensions of the formula have been obtained. The Giambelli problem consists in finding a "closed formula" for a Schubert class in terms of those special classes, and in the torus equivariant setting it is closely related to the theory of degeneracy loci of vector bundles ( $c f$. [FP98], [Tam] and the references therein).

For the symplectic or orthogonal Grassmannians, there is a natural notion of special Schubert classes, which takes into account the isotropic conditions arising from the symplectic or orthogonal form. For the Grassmannian of maximal isotropic subspaces, the Giambelli formula, first found by Pragacz [Pra91], expresses a general Schubert class as a Pfaffian whose entries are appropriate quadratic polynomials in the special Schubert classes. Its natural equivariant version was obtained by Kazarian [Kaz00] in the context of degeneracy loci, and later it was also proved in [Ike07] and [IN09] in terms of equivariant cohomology by using more algebraic methods. Similarly to Pragacz's formula, also Kazarian's expression

\footnotetext{
${ }^{\dagger}$ Email: ikeexmath.ous.ac.jp

‡Email: tomoomatsumura@kaist.ac.kr

1365-8050 @ 2015 Discrete Mathematics and Theoretical Computer Science (DMTCS), Nancy, France
} 
is given as a single Pfaffian. On the other hand, for the non-maximal isotropic Grassmannians, an answer to the (non-equivariant) Giambelli problem was given by Buch, Kresch, and Tamvakis [BKTb, BKTa]. Their formula expresses an arbitrary Schubert class as a polynomial in the special Schubert classes, it is defined by means of Young's raising operators. We can regard this polynomial expression as a certain "combinatorial interpolation" between the Jacobi-Trudi determinant and the Schur Pfaffian.

This paper is mainly concerned with the equivariant Giambelli problem for the non-maximal isotropic Grassmannians in the symplectic case. In [Wil10], Wilson employed the raising operators to define the double theta polynomials, and proved that these polynomials satisfy the equivariant Chevalley formula for the non-maximal symplectic Grassmannian. In [Wil10], it was further conjectured that the double theta polynomials would be equal to the double Schubert polynomials in [IMN11], a distinguished family of polynomials which are identified with the equivariant Schubert classes of the flag variety in the infinite rank setting.

The main result of this paper provides a formula expressing each double Schubert polynomial associated to the isotropic Grassmannians as a sum of Pfaffians whose entries are Wilson's double theta polynomials corresponding to the special Schubert classes. This immediately leads to a proof of Wilson's conjecture, because the raising operator formula can be rewritten as a Pfaffian sum by a formal computation. Our method to prove the main result is to use the left divided difference operators that are essential in the theory of (double) Schubert polynomials and exist only in its equivariant setup.

This article is a short version of the paper [IM15]. Detailed proofs are omitted. The only content which is not included in [IM15] is about a combinatorial description of the Bruhat order on a certain parabolic quotient of the type $C$ Weyl group (Theorem 2.8.

\section{Double Schubert polynomials of type $C$}

We first recall the definition and fundamental properties of the double Schubert polynomials introduced in [IMN11]. Then we discuss the correspondences among the $k$-strict partitions, the characteristic indices and the parabolic quotients of the Weyl group.

\subsection{Double Schubert polynomials}

Weyl group for type C Let $W_{\infty}$ be the infinite hyperoctahedral group which is defined by the generators $s_{i}, i=0,1, \ldots$, and the relations

$$
\begin{gathered}
s_{i}^{2}=e \quad(i \geq 0), \quad s_{i} s_{j}=s_{j} s_{i} \quad(|i-j| \geq 2), \\
s_{0} s_{1} s_{0} s_{1}=s_{1} s_{0} s_{1} s_{0}, \quad s_{i} s_{i+1} s_{i}=s_{i+1} s_{i} s_{i+1} \quad(i \geq 1) .
\end{gathered}
$$

We identify $W_{\infty}$ with the group of all permutations $w$ of $\mathbb{Z} \backslash\{0\}$ such that $w(i) \neq i$ for only finitely many $i \in \mathbb{Z} \backslash\{0\}$, and $\overline{w(i)}=w(\bar{i})$ for all $i$ where $\bar{i}:=-i$. The generators, often referred to as the simple reflections, are identified with the transpositions $s_{0}=(1, \overline{1})$ and $s_{i}=(i+1, i)(\bar{i}, \overline{i+1})$ for $i \geq 1$. Let $W_{n}$ be the subgroup of $W_{\infty}$ generated by $s_{0}, s_{1}, \ldots, s_{n-1}$. Or equivalently, it consists of elements $w \in W_{\infty}$ such that $w(i)=i$ for all $i>n$. The one-line notation of an element $w \in W_{\infty}$ is the sequence $w=(w(1) w(2) w(3) \cdots)$. We often write the one-line notation of $w \in W_{n}$ by the finite sequence $(w(1) w(2) \cdots w(n))$. The length of $w \in W_{\infty}$ is denoted by $\ell(w)$. 
The ring of Double Schubert Polynomials for type C Let $x=\left(x_{1}, x_{2}, \ldots\right)$ be a sequence of indeterminates. Let $Q_{r}(x)(r \geq 0)$ be the functions defined by the generating function

$$
\sum_{r=0}^{\infty} Q_{r}(x) u^{r}=\prod_{i=1}^{\infty} \frac{1+x_{i} u}{1-x_{i} u}
$$

Let $\Gamma$ be the ring generated by $Q_{r}(x)(r \geq 0)$. Let $\mathcal{R}_{\infty}$ be the polynomial ring $\Gamma[t, z]$ in the indeterminates $t=\left(t_{1}, t_{2}, \ldots\right)$, and $z=\left(z_{1}, z_{2}, \ldots\right)$ with the coefficients in $\Gamma$.

There is a distinguished $\mathbb{Z}[t]$-basis $\mathfrak{C}_{w}\left(w \in W_{\infty}\right)$ of $\mathcal{R}_{\infty}$ called the double Schubert polynomials of type $C$. To characterize this basis, we first define two actions of $W_{\infty}$ on $\mathcal{R}_{\infty}$ (the right and left actions), and then introduce the right and left divided difference operators.

The right action on $\mathcal{R}_{\infty}$ as a $\mathbb{Z}[t]$-algebra For each $i \geq 1$, define the $\mathbb{Z}[t]$-algebra automorphism $s_{i}^{z}$ of $\mathcal{R}_{\infty}$ by

$$
s_{i}^{z}\left(z_{i}\right)=z_{i+1}, \quad s_{i}^{z}\left(z_{i+1}\right)=z_{i}, \quad s_{i}^{z}\left(z_{j}\right)=z_{j}(j \neq i, i+1), \quad \text { and } \quad s_{i}^{z}\left(Q_{r}(x)\right)=Q_{r}(x) .
$$

Also define the $\mathbb{Z}[t]$-algebra automorphism $s_{0}^{z}$ of $\mathcal{R}_{\infty}$ by

$$
s_{0}^{z}\left(z_{1}\right)=-z_{1}, \quad s_{0}^{z}\left(z_{i}\right)=z_{i}(i \geq 1), \quad \text { and } \quad s_{0}^{z} Q_{r}\left(x_{1}, x_{2}, \ldots\right)=Q_{r}\left(z_{1}, x_{1}, x_{2}, \ldots\right) .
$$

These satisfy the Coxeter relations $\left[2.1\right.$, and therefore define an action of $W_{\infty}$ on $\mathcal{R}_{\infty}$ as a $\mathbb{Z}[t]$-algebra.

The left action on $\mathcal{R}_{\infty}$ as a $\mathbb{Z}[z]$-algebra Consider the involution $\omega$ of the ring $\mathcal{R}_{\infty}$ :

$$
\omega\left(t_{i}\right)=-z_{i}, \omega\left(z_{i}\right)=-t_{i}, \omega\left(Q_{r}(x)\right)=Q_{r}(x) .
$$

For each $i \geq 0$, we define the $\mathbb{Z}[z]$-algebra automorphism $s_{i}^{t}$ of $\mathcal{R}_{\infty}$ by $s_{i}^{t}:=\omega s_{i}^{z} \omega$. In particular, we have

$$
s_{0}^{t} Q_{r}\left(x_{1}, x_{2}, \ldots\right)=Q_{r}\left(-t_{1}, x_{1}, x_{2}, \ldots\right) .
$$

These satisfy the Coxeter relations 2.1 , and therefore define an action of $W_{\infty}$ on $\mathcal{R}_{\infty}$ as a $\mathbb{Z}[z]$-algebra.

Divided difference operators We define the right and left divided difference operators respectively by

$$
\partial_{i} f=\frac{f-s_{i}^{z} f}{\omega\left(\alpha_{i}\right)} \quad \text { and } \quad \delta_{i} f=\frac{f-s_{i}^{t} f}{\alpha_{i}} \quad\left(i \geq 0, f \in \mathcal{R}_{\infty}\right),
$$

where $\alpha_{i}(i \geq 0)$ are the simple roots defined by $\alpha_{0}=2 t_{1}$ and $\alpha_{i}=t_{i+1}-t_{i}$ for all $i \geq 1$.

Theorem 2.1 ([IMN11]) There exists a unique $\mathbb{Z}[t]$-free basis $\left\{\mathfrak{C}_{w}(z, t ; x) \mid w \in W_{\infty}\right\}$ of $\mathcal{R}_{\infty}$ satisfying the equations

$$
\partial_{i} \mathfrak{C}_{w}=\left\{\begin{array}{ll}
\mathfrak{C}_{w s_{i}} & \text { if } \ell\left(w s_{i}\right)<\ell(w) \\
0 & \text { otherwise }
\end{array} \quad \text { and } \quad \delta_{i} \mathfrak{C}_{w}= \begin{cases}\mathfrak{C}_{s_{i} w} & \text { if } \ell\left(s_{i} w\right)<\ell(w) \\
0 & \text { otherwise }\end{cases}\right.
$$

for all $i \geq 0$, and such that $\mathfrak{C}_{w}$ has no constant term except $\mathfrak{C}_{e}=1$. 


\subsection{The $k$-Grassmannian elements and the invariant subring $\mathcal{R}_{\infty}^{(k)}$}

Let $W_{(k)}$ be the subgroup of $W_{\infty}$ generated by all $s_{i}, i \neq k$. Let $W_{\infty}^{(k)}$ be the set of minimum length coset representatives for $W_{\infty} / W_{(k)}$, and it is given by

$$
W_{\infty}^{(k)}=\left\{w \in W_{\infty} \mid \ell\left(w s_{i}\right)>\ell(w) \text { for all } i \neq k\right\} .
$$

We denote $W_{\infty}^{(k)} \cap W_{n}$ by $W_{n}^{(k)}$. An element of $W_{\infty}^{(k)}$ is called $k$-Grassmannian and it is given by the following one-line notation:

$$
\begin{gathered}
w=\left(v_{1} \cdots v_{k} \mid \overline{\zeta_{1}} \cdots \overline{\zeta_{s}} u_{1} u_{2} \cdots\right) \\
0<v_{1}<\cdots<v_{k}, \overline{\zeta_{1}}<\cdots<\overline{\zeta_{s}}<0<u_{1}<u_{2}<\cdots .
\end{gathered}
$$

We insert a vertical line after $v_{k}$ to indicate that $w$ is regarded as a $k$-Grassmannian element. For example, $(13 \mid \overline{4} 256 \cdots)$ is a 2 -Grassmannian element in $W_{\infty}$.

Lemma 2.2 Let $w \in W_{\infty}^{(k)}$. We have $\ell\left(s_{0} w\right)<\ell(w)$ if and only if

(L0) $w=(\cdots \mid \cdots \overline{1} \cdots)$, i.e., $\zeta_{s}=1$.

Suppose $i \geq 1$. Then $\ell\left(s_{i} w\right)<\ell(w)$ if and only if one of the following holds:

(L1) $w=(\cdots \mid \cdots \overline{i+1} \cdots i \cdots)$, i.e., $\zeta_{p}=i+1$ and $u_{q}=i$ for some $p$ and $q$;

(L2) $w=(\cdots i \cdots \mid \cdots \overline{i+1} \cdots)$, i.e., $\zeta_{p}=i+1$ and $v_{q}=i$ for some $p$ and $q$;

(L3) $w=(\cdots i+1 \cdots \mid \cdots i \cdots)$, i.e., $u_{p}=i$ and $v_{q}=i+1$ for some $p$ and $q$.

In particular, for any $i \in\{0,1,2, \ldots\},, \ell\left(s_{i} w\right)=\ell(w)-1$ implies $s_{i} w \in W_{\infty}^{(k)}$.

Let $\mathcal{R}_{\infty}^{(k)}$ be the $\mathbb{Z}[t]$-subalgebra of $\mathcal{R}_{\infty}$ fixed by the right action of $W_{(k)}$ :

$$
\mathcal{R}_{\infty}^{(k)}:=\left\{f \in \mathcal{R}_{\infty} \mid s_{i}^{z}(f)=f \quad(\forall i \neq k)\right\} .
$$

We can show that $\mathcal{R}_{\infty}^{(k)}=\bigoplus_{w \in W_{\infty}^{(k)}} \mathbb{Z}[t] \mathfrak{C}_{w}$. Furthermore, the double Schubert polynomials $\mathfrak{C}_{w}$ for the $k$-Grassmannian elements $w \in W_{\infty}^{(k)}$ are characterized in terms of the left divided difference operators as follows. For $f \in \mathcal{R}_{\infty}$, let $\left.f\right|_{\emptyset}$ denote the polynomial in $\mathbb{Z}[t]$ obtained from $f$ by the substitutions $x_{i} \mapsto 0$ and $z_{i} \mapsto t_{i}$ for all $i$.

Proposition 2.3 If a family $F_{w}, w \in W_{\infty}^{(k)}$ of elements of $\mathcal{R}_{\infty}^{(k)}$ satisfies the following conditions

$$
\begin{gathered}
\delta_{i} F_{w}= \begin{cases}F_{s_{i} w} & \text { if } \ell\left(s_{i} w\right)<\ell(w) \\
0 & \text { if } \ell\left(s_{i} w\right)>\ell(w)\end{cases} \\
\left.F_{w}\right|_{\emptyset}=\delta_{w, e},
\end{gathered}
$$

then $F_{w}=\mathfrak{C}_{w}$ for all $w \in W_{\infty}^{(k)}$. 


\subsection{The $k$-strict partitions}

A partition $\lambda$ is a sequence of non-increasing non-negative integers $\left(\lambda_{1}, \lambda_{2}, \ldots\right)$ where only finitely many $\lambda_{i}$ 's are positive. It is called $k$-strict if no part greater than $k$ is repeated. Let $\mathcal{P}_{\infty}^{(k)}$ be the set of all $k$ strict partitions. Let $\mathcal{P}_{n}^{(k)} \subset \mathcal{P}_{\infty}^{(k)}$ be the subset of all $k$-strict partitions whose Young diagrams fit in the $(n-k) \times(n+k)$ rectangle. We often denote $\lambda$ by a finite sequence $\left(\lambda_{1}, \ldots, \lambda_{r}\right)$, assuming $\lambda_{i}=0$ for all $i>r$, and also denote the sum of the positive entries by $|\lambda|$.

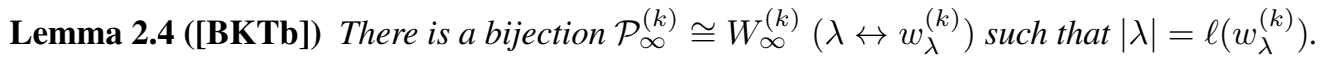

We describe the bijection below. Let $\lambda=\left(\lambda_{1}, \lambda_{2}, \ldots\right) \in \mathcal{P}_{\infty}^{(k)}$. Let $s$ be an index such that $\lambda_{s}>k$ and $\lambda_{s+1} \leq k$. Let $\zeta_{i}:=\lambda_{i}-k$ for $i \in\{1, \ldots, s\}$. Let $\mu=\left(\mu_{1}, \ldots, \mu_{k}\right)$ be the conjugate of the partition $\left(\lambda_{s+1}, \lambda_{s+2}, \ldots\right)$. For $i \in\{1, \ldots, k\}$, define

$$
v_{i}=s+\mu_{k+1-i}+i-\sharp\left\{p \mid \zeta_{p}>s+\mu_{k+1-i}+i\right\} .
$$

The signed permutation $w_{\lambda}$ is given by the one-line notation 2.4 where $u_{1}, u_{2}, \ldots$ are the increasing positive integers determined uniquely by $v$ and $\zeta$.

Conversely, let $w \in W_{\infty}^{(k)}$ with the one-line notation 2.4 . Define a partition $\nu=\left(\nu_{1}, \nu_{2}, \ldots\right)$ by $\nu_{i}=\sharp\left\{p \mid v_{p}>u_{i}\right\}$. Then a $k$-strict partition $\lambda$ defined by setting $\lambda_{i}=\zeta_{i}+k$ if $1 \leq i \leq s$ and $\lambda_{i}=\nu_{i-s}$ if $s+1 \leq i$.

Remark 2.5 By the bijection in Lemma 2.4 the natural Bruhat order on $W_{\infty}^{(k)}$ does not correspond to the order on $\mathcal{P}_{\infty}^{(k)}$ defined by inclusions of Young diagrams. For example, consider 1-strict partitions $\lambda=(21)$ and $\mu=(4)$. The corresponding permutations are $w_{\lambda}^{(1)}=(3 \overline{1} 24)=s_{2} s_{0} s_{1}$ and $w_{\mu}^{(1)}=$ $(1 \overline{3} 24)=s_{2} s_{1} s_{0} s_{1}$ respectively. We see that $w_{\lambda}^{(1)} \leq w_{\mu}^{(1)}$, while $\lambda \not \subset \mu$.

\subsection{Characteristic index and Bruhat order on $W_{\infty}^{(k)}$}

The following indexing set for $W_{\infty}^{(k)}$ turns out to be useful for both the understanding of Bruhat order and the computation in the left divided difference operators.

Definition 2.6 (Characteristic index) For each $w \in W_{\infty}^{(k)}$ with the one-line notation (2.4), we define the associated characteristic index by

$$
\chi=\left(\chi_{1}, \chi_{2}, \ldots\right)=\left(\zeta_{1}-1, \zeta_{2}-1, \ldots, \zeta_{s}-1,-u_{1},-u_{2}, \ldots\right)
$$

In particular, if $w \in W_{n}^{(k)}$, then $\chi_{i}=-i-k$ for all $i>n-k$.

If $\chi$ and $\lambda$ are the characteristic index and the $k$-strict partition associated to $w$ respectively, then we have $\chi_{j}=\lambda_{j}-k-j+\sharp\left\{i \mid i<j, \lambda_{i}+\lambda_{j}>2 k+j-i\right\}$.

For a strictly decreasing sequence $\chi=\left(\chi_{1}, \chi_{2}, \ldots\right)$ of integers, let

$$
b_{i}^{\chi}=\left\{\begin{array}{ll}
\chi_{i}+1 & \text { if } \chi_{i} \geq 0 \\
-\chi_{i} & \text { if } \chi_{i}<0,
\end{array} \quad \text { for all } i=1,2, \ldots\right.
$$


Definition 2.7 Let $\Xi_{\infty}^{(k)}$ be the set of all strictly decreasing sequences $\chi=\left(\chi_{1}, \chi_{2}, \ldots\right)$ of integers such that $b_{i}^{\chi}$ 's are all distinct, $\{1,2, \ldots\} \backslash\left\{b_{i}^{\chi}, i=1,2, \ldots\right\}$ is a finite set of cardinality $k$, and $\chi_{i}=-i-k$ for sufficiently large $i$. Let $\Xi_{n}^{(k)}$ be the subset of $\Xi_{\infty}^{(k)}$ consisting of $\chi$ such that $\chi_{i}=-i-k$ for all $i>n-k$. Define a partial order on $\Xi_{\infty}^{(k)}$ by

$$
\chi \leq \chi^{\prime} \quad \text { if and only if } \quad \chi_{i} \leq \chi_{i}^{\prime} \text { all } i=1,2, \ldots
$$

Theorem 2.8 The map $W_{\infty}^{(k)} \rightarrow \Xi_{\infty}^{(k)}$ associating the characteristic indices is an order preserving bijection. Furthermore this map also induces an order preserving bijection $W_{n}^{(k)} \cong \Xi_{n}^{(k)}$.

The proof of this theorem is postponed until Section 3.3 .

Example 2.9 Let $\lambda$ and $\mu$ be the partitions given in Remark 2.5 The corresponding characteristic indices are $\chi_{\lambda}=(0,-2,-4)$ and $\chi_{\mu}=(2,-2,-4)$ respectively. We find $\chi_{\lambda} \leq \chi_{\mu}$. This verifies Theorem 2.8 since we know $w_{\lambda} \leq w_{\mu}$ from Remark 2.5 .

\section{Equivariant Schubert classes of symplectic Grassmannians}

We explain the fact that the double Schubert polynomials represent the equivairant Schubert classes.

\subsection{Flag varieties of type $C$}

Let $G=\operatorname{Sp}_{2 n}(\mathbb{C})$ be the complex symplectic group of rank $n$. Let $B$ be a Borel subgroup of $G$, and $T$ the maximal torus contained in $B$ (see [IMN11] for the precise conventions). The flag variety $\mathcal{F} l_{n}$ is defined as the quotient space $G / B$. The Weyl group $N_{G}(T) / T$ is identified with $W_{n}$. For each $w \in W_{n}$, there is the point $e_{w}$ in $\mathcal{F} l_{n}$. Let $B^{-}$be the Borel subgroup such that $B \cap B^{-}=T$. The Schubert variety $X_{w}$ is defined as the Zariski closure of $B^{-}$-orbit of the point $e_{w} \in \mathcal{F} l_{n}$. The codimension of $X_{w}$ is precisely the length $\ell(w)$ of $w$. Since $X_{w}$ is a $T$-stable subvariety of $\mathcal{F} l_{n}$ it defines the fundamental class $\left[X_{w}\right]_{T}$ in the integral $T$-equivariant cohomology ring $H_{T}^{*}\left(\mathcal{F} l_{n}\right)$. We call $\left[X_{w}\right]_{T}$ the equivariant Schubert class associated to $w \in W_{n}$. We regard $H_{T}^{*}\left(\mathcal{F} l_{n}\right)$ as a $\mathbb{Z}[t]$-algebra by identifying $H_{T}^{*}(p t)=\mathbb{Z}\left[t_{1}, \ldots, t_{n}\right]$ and defining the action of $t_{i}$ to be trivial for all $i>n$.

Theorem 3.1 ([IMN11]) There is a canonical $\mathbb{Z}[t]$-algebra homomorphism $\pi_{n}: \mathcal{R}_{\infty} \longrightarrow H_{T}^{*}\left(\mathcal{F} l_{n}\right)$ such that $\pi_{n}$ sends $\mathfrak{C}_{w}(z, t ; x)$ to $\left[X_{w}\right]_{T}$ if $w \in W_{n}$ and to zero if $w \notin W_{n}$.

\subsection{Symplectic Grassmannian and its Schubert varieties}

We fix a non-negative integer $k$. For any positive integer $n \geq k$, let $\mathrm{SG}_{n}^{k}$ denote the Grassmannian of $(n-k)$-dimensional isotropic subspaces in $\mathbb{C}^{2 n}$ equipped with a symplectic form. There is a maximal parabolic subgroup $P_{k}$ of the symplectic group $G=\mathrm{Sp}_{2 n}(\mathbb{C})$ such that $\mathrm{SG}_{n}^{k}$ can be realized as the homogeneous space $G / P_{k}$.

Let $\mathbf{e}_{1}, \ldots, \mathbf{e}_{n}, \mathbf{e}_{1}^{*}, \ldots, \mathbf{e}_{n}^{*}$ be a standard symplectic basis of $\mathbb{C}^{2 n}$, i.e., define the symplectic form by $\left\langle\mathbf{e}_{i}, \mathbf{e}_{j}\right\rangle=\left\langle\mathbf{e}_{i}^{*}, \mathbf{e}_{j}^{*}\right\rangle=0,\left\langle\mathbf{e}_{i}, \mathbf{e}_{j}^{*}\right\rangle=\delta_{i j}$. For $1 \leq i \leq n$, define a complete flag

$$
F^{\bullet}: F^{n} \subset \cdots \subset F^{1} \subset F^{\overline{1}} \subset \cdots \subset F^{\bar{n}}
$$


by $F^{i}=\left\langle\mathbf{e}_{i}, \ldots, \mathbf{e}_{n}\right\rangle$ and $F^{\bar{i}}=\left\langle\mathbf{e}_{i}^{*}, \ldots, \mathbf{e}_{1}^{*}\right\rangle+F^{1}$. For each $\lambda \in \mathcal{P}_{n}^{(k)}$, the corresponding Schubert variety $\Omega_{\lambda}$ with respect to $F^{\bullet}$ can be defined as

$$
\Omega_{\lambda}:=\left\{V \in \mathrm{SG}_{n}^{k} \mid \operatorname{dim}\left(V \cap \overline{F_{\lambda}^{(k)}(k+j)}\right) \geq j \quad(1 \leq j \leq n-k)\right\} .
$$

In particular, the special Schubert varieties are the ones associated to one-line $k$-strict partitions:

$$
\Omega_{r}=\left\{V \in \mathrm{SG}_{n}^{k} \mid \operatorname{dim}\left(V \cap F^{r-k-1}\right) \geq 1\right\},
$$

for $1 \leq r \leq n+k$. Their classes $\left[\Omega_{r}\right]_{T}$ are called the special Schubert classes. They are equal to the $r$-th equivariant Chern classes $c_{r}(\mathcal{Q})$ of the universal quotient bundle $\mathcal{Q}$ over $\mathrm{SG}_{n}^{k}$. For $1 \leq j \leq n-k$, we have

$$
\operatorname{dim} F^{\overline{w_{\lambda}^{(k)}(k+j)}}=n-\chi_{j} .
$$

This explains how naturally the characteristic index is defined.

There is the following commutative diagram :

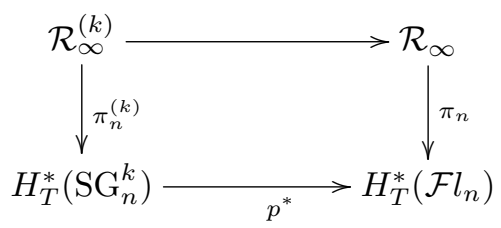

where the horizontal arrow $p^{*}$ in the second row is the pullback of the natural projection $p: \mathcal{F} l_{n} \rightarrow \mathrm{SG}_{n}^{k}$ and $\pi_{n}^{(k)}$ is obtained by restricting $\pi_{n}$ to $\mathcal{R}_{\infty}^{(k)}$. If $\lambda \in \mathcal{P}_{n}^{(k)}$, we have $p^{*}\left[\Omega_{\lambda}\right]_{T}=\left[X_{w_{\lambda}^{(k)}}\right]_{T}$ and

$$
\pi_{n}^{(k)}\left(\mathfrak{C}_{w_{\lambda}^{(k)}}(z, t ; x)\right)=\left[\Omega_{\lambda}\right]_{T}
$$

In particular, the special Schubert class $\left[\Omega_{r}\right]_{T}$ of degree $r$ is the image of $\mathfrak{C}_{w_{r}^{(k)}}(z, t ; x)$ where $w_{r}^{(k)}$ is the element of $W_{\infty}^{(k)}$ corresponding to the partition with $r$ boxes in one row.

\subsection{Proof of Theorem 2.8}

By the definition of the characteristic index, it is clear that the map is a bijection. Choose $n$ such that $w, w^{\prime} \in W_{n}^{(k)}$. Let $\chi$ and $\chi^{\prime}$ be the characteristic indices of $w$ and $w^{\prime}$ respectively. Let $\lambda$ and $\lambda^{\prime}$ be the strict partitions corresponding to $w$ and $w^{\prime}$ respectively. We reindex the flag $F^{\bullet}$ defined in 3.1 , and consider the complete flag $U^{\bullet}: 0=U^{n} \subset \cdots U^{n-1} \cdots \subset U^{0} \subset U^{-1} \subset \cdots \subset U^{-n}=\mathbb{C}^{2 n}$ where for each $i \in\{1,2, \ldots, n\}, U^{-i}=F^{\bar{i}}$ and $U^{i}=F^{i-1}$. Note that $\operatorname{dim} U^{i}=n-i$ for all $-n \leq i \leq n$ by (3.3. Then the Schubert variety $\Omega_{\lambda}$ is given by

$$
\Omega_{\lambda}=\left\{V \in \mathrm{SG}_{n}^{k} \mid \operatorname{dim}\left(V \cap U^{\chi_{i}}\right) \geq i, i=1, \ldots, n-k\right\} .
$$

It is well-known that $\Omega_{\lambda} \supset \Omega_{\lambda^{\prime}}$ if and only if $w \leq w^{\prime}$. Therefore $w \leq w^{\prime}$ if and only if, for all $i=1, \ldots, n-k$, we have

$$
U^{\chi_{i}^{\prime}} \subset U^{\chi_{i}} \text { i.e. } \chi_{i} \leq \chi_{i}^{\prime}
$$




\section{Pfaffian sum formula for the equivariant Schubert classes}

\subsection{Double theta polynomials}

Our goal is to give an explicit closed formula to describe $\mathfrak{C}_{w}(z, t ; x)\left(w \in W_{\infty}^{(k)}\right)$ as a polynomial in terms of the double Schubert polynomials $\mathfrak{C}_{w_{r}}(z, t ; x)$ corresponding to the special classes $\left[\Omega_{r}\right]_{T}$.

Definition 4.1 Define ${ }_{k} \vartheta_{r}^{(l)}(x, z \mid t)$ for $l, r \geq 0$ by

$$
\begin{aligned}
\sum_{r=0}^{\infty}{ }_{k} \vartheta_{r}^{(l)}(x, z \mid t) \cdot u^{r} & =\prod_{i=1}^{\infty} \frac{1+x_{i} u}{1-x_{i} u} \prod_{i=1}^{k}\left(1+z_{i} u\right) \prod_{i=1}^{l}\left(1-t_{i} u\right) \\
\sum_{r=0}^{\infty}{ }_{k} \vartheta_{r}^{(-l)}(x, z \mid t) \cdot u^{r} & =\prod_{i=1}^{\infty} \frac{1+x_{i} u}{1-x_{i} u} \prod_{i=1}^{k}\left(1+z_{i} u\right) \prod_{i=1}^{l} \frac{1}{1+t_{i} u}
\end{aligned}
$$

For $r<0$, we set ${ }_{k} \vartheta_{r}^{(l)}(x, z \mid t)=0$. We omit $k$ when it is made clear by the context. Under $\pi_{n}$, the indeterminates $z_{i}$ correspond to the Chern roots of the tautological bundles and those theta polynomials ${ }_{k} \vartheta_{r}^{(l)}(x, z \mid t)$ map to Chern classes of certain virtual bundles. In particular, Wilson in [Will0, Prop. 6] proved

$$
\mathfrak{C}_{w_{r}}(z, t ; x)={ }_{k} \vartheta_{r}^{(r-k-1)}(x, z \mid t)
$$

Although the above definition of the double theta polynomials appears slightly different from the one in Wilson's thesis [Wil10], one recovers Wilson's definition after applying appropriate changes of indices. See [IM15] for details. We can prove the following lemma from the definitions by direct computations.

Lemma 4.2 (a) For all $i \geq 0$, we have $\delta_{i} \vartheta_{r}^{(l)}=0$ if $l \neq \pm i$ and $\delta_{i} \vartheta_{r}^{(l)}=\vartheta_{r-1}^{(l-1)}$ if $l= \pm i$.

(b) For $i \neq 0$, we have $\delta_{i}\left(\vartheta_{r}^{(i)} \cdot \vartheta_{s}^{(-i)}\right)=\vartheta_{r-1}^{(i-1)} \cdot \vartheta_{s}^{(-i-1)}+\vartheta_{r}^{(i-1)} \cdot \vartheta_{s-1}^{(-i-1)}$.

\subsection{Multi Schur-Pfaffian}

For each $i \geq 1$, let $c^{(i)}$ be an infinite sequence of indeterminates $c_{r}^{(i)}, r \in \mathbb{Z}$. Consider the polynomial ring $\mathbb{Z}[c]:=\mathbb{Z}\left[c_{r}^{(i)}, i \geq 1, r \in \mathbb{Z}\right]$. The multi Schur-Pfaffian

$$
\operatorname{Pf}\left[c_{r_{1}}^{(1)} \ldots c_{r_{m}}^{(m)}\right] \in \mathbb{Z}[c]
$$

is defined as follows:

- for $m=1$ we set $\operatorname{Pf}\left[c_{r}^{(1)}\right]=c_{r}^{(1)}$.

- for $m=2$, we set $\operatorname{Pf}\left[c_{r_{1}}^{(1)} c_{r_{2}}^{(2)}\right]=c_{r_{1}}^{(1)} c_{r_{2}}^{(2)}+2 \sum_{s=1}^{r_{2}}(-1)^{s} c_{r_{1}+s}^{(1)} c_{r_{2}-s}^{(2)}$.

- for any odd $m \geq 3$, we set $\operatorname{Pf}\left[c_{r_{1}}^{(1)} \cdots c_{r_{m}}^{(m)}\right]=\sum_{s=1}^{m}(-1)^{s-1} c_{r_{s}}^{(s)} \cdot \operatorname{Pf}\left[c_{r_{1}}^{(1)} \cdots \widehat{c_{r_{s}}^{(s)}} \cdots c_{r_{m}}^{(m)}\right]$.

- for any even $m \geq 4$, we set $\operatorname{Pf}\left[c_{r_{1}}^{(1)} \ldots c_{r_{m}}^{(m)}\right]=\sum_{s=2}^{m}(-1)^{s} \operatorname{Pf}\left[c_{r_{1}}^{(1)} c_{r_{s}}^{(s)}\right] \cdot \operatorname{Pf}\left[c_{r_{2}}^{(2)} \ldots \widehat{c_{r_{s}}^{(s)}} \ldots c_{r_{m}}^{(m)}\right]$.

The following properties follow from the above definition of Pfaffian by the induction on $m$. 


\section{Proposition 4.3}

(1) If $\operatorname{Pf}\left[c_{r}^{(l)} c_{r}^{(l)}\right]=0$, then we have $\operatorname{Pf}\left[c_{r_{1}}^{\left(l_{1}\right)} \cdots c_{r}^{(l)} c_{r}^{(l)} \cdots c_{r_{m}}^{\left(l_{m}\right)}\right]=0$.

(2) If $\operatorname{Pf}\left[c_{r}^{(l)} c_{s}^{(l)}\right]+\operatorname{Pf}\left[c_{s}^{(l)} c_{r}^{(l)}\right]=0$, then we have

$$
\operatorname{Pf}\left[c_{r_{1}}^{\left(l_{1}\right)} \ldots c_{r}^{(l)} c_{s}^{(l)} \cdots c_{r_{m}}^{\left(i_{m}\right)}\right]+\operatorname{Pf}\left[c_{r_{1}}^{\left(l_{1}\right)} \ldots c_{s}^{(l)} c_{r}^{(l)} \cdots c_{r_{m}}^{\left(l_{m}\right)}\right]=0 .
$$

\subsection{Pfaffian sum formula (Main Theorem)}

Definition 4.4 For all $\left(r_{1}, \ldots, r_{m}\right),\left(l_{1}, \ldots, l_{m}\right) \in \mathbb{Z}^{m}$, let

$$
\operatorname{Pf}\left[\vartheta_{r_{1}}^{\left(l_{1}\right)} \vartheta_{r_{2}}^{\left(l_{2}\right)} \cdots \vartheta_{r_{m}}^{\left(l_{m}\right)}\right]:=\left.\operatorname{Pf}\left[c_{r_{1}}^{(1)} c_{r_{2}}^{(2)} \cdots c_{r_{m}}^{(m)}\right]\right|_{c=\vartheta^{(l)}}
$$

where $\left.\right|_{c=\vartheta^{(l)}}$ means that we substitute $\vartheta_{s}^{\left(l_{i}\right)}$ for $c_{s}^{(i)}$ for all $i \in\{1, \ldots, m\}$ and $s \in \mathbb{Z}$.

Let $\Delta=\{(i, j) \mid 1 \leq i<j \leq n-k\}$. Define a subset $D(\lambda)$ of $\Delta$ by $D(\lambda):=\left\{(i, j) \in \Delta \mid \chi_{i}+\chi_{j}<\right.$ $0\}$. The main result of this paper is the following.

Theorem 4.5 (Main Theorem) Let $\lambda$ be a k-strict partition in $\mathcal{P}_{n}^{(k)}$. We have

$$
\mathfrak{C}_{w_{\lambda}^{(k)}}^{(k)} \sum_{I \subset D(\lambda)} \operatorname{Pf}\left[\vartheta_{\lambda_{1}+a_{1}^{I}}^{\left(\chi_{1}\right)} \cdots \vartheta_{\lambda_{n-k}+a_{n-k}^{I}}^{\left(\chi_{n-k}\right)}\right]
$$

where I runs over all subsets of $D(\lambda)$ and $a_{s}^{I}=\#\{j \mid(s, j) \in I\}-\#\{i \mid(i, s) \in I\}$.

Example 4.6 Let $k=1, n=5$. Let $\lambda=(5,3,2,1)$ be a $k$-strict partition. Then $w_{\lambda}^{(1)}=5 \mid \overline{4} \overline{2} \overline{1} 3$ and $D(\lambda)=\{(2,4),(3,4)\}$. We have

$$
\mathfrak{C}_{w_{\lambda}^{(1)}}=\operatorname{Pf}\left[\vartheta_{5}^{(3)} \vartheta_{3}^{(1)} \vartheta_{2}^{(0)} \vartheta_{1}^{(-3)}\right]+\operatorname{Pf}\left[\vartheta_{5}^{(3)} \vartheta_{4}^{(1)} \vartheta_{2}^{(0)} \vartheta_{0}^{(-3)}\right]+\operatorname{Pf}\left[\vartheta_{5}^{(3)} \vartheta_{3}^{(1)} \vartheta_{3}^{(0)} \vartheta_{0}^{(-3)}\right] .
$$

Once we read the formula in terms of raising operators, the following corollary is immediate.

Corollary 4.7 If $D(\lambda)=\Delta$, in particular, if $\lambda$ is contained in the $(n-k) \times k$ rectangle, then $\mathfrak{C}_{w_{\lambda}^{(k)}}$ is a single determinant $\operatorname{det}\left(\vartheta_{\lambda_{i}+j-i}^{\left(\chi_{i}\right)}\right)_{1 \leq i, j \leq n-k}([$ [Tam $\S 1])$. If $D(\lambda)=\emptyset$, in particular, if $\lambda$ is a strict partition containing the $(n-k) \times k$ rectangle, then $\mathfrak{C}_{w_{\lambda}^{(k)}}$ is a single Pfaffian $\operatorname{Pf}\left[\vartheta_{\lambda_{1}}^{\left(\chi_{1}\right)} \cdots \vartheta_{\lambda_{n-k}}^{\left(\chi_{n-k}\right)}\right]$.

In the case when $\lambda$ is contained in the $(n-k) \times k$ rectangle, the result was proved by Wilson [Willo].

\subsection{Related results}

Anderson and Fulton $[\mathrm{AF}]$ defined a notion of vexillary signed permutation in type B,C, and D. They showed the double Schubert polynomials associated to vexillary signed permutations are given by explicit Pfaffian formulas. Naruse [ $\overline{\mathrm{Nar}}]$ also independently proved a formula that expresses the corresponding double Schubert polynomials as a specialization of the factorial $Q$ - and $P$-functions. Since our formula also expresses some Schubert classes as single Pfaffians, there is an overlap between our results and the results of [AF]. However, not all $k$-Grassmannian permutations are vexillary and there are non-vexillary $k$-Grassmannian permutations whose corresponding classes are written as single Pfaffians, e.g. $13 \mid \overline{5} \overline{4} 2$ is not vexillary but $\mathfrak{C}_{13 \mid \overline{5} \overline{4} 2}$ is a single Pfaffian as above. 


\section{Proof of Main Theorem}

\subsection{Key results on multi Schur-Pfaffian with entries $\vartheta_{r}^{(l)}$}

The proof of our main theorem is essentially based on the following two propositions. We can show them by using the inductive definition of the multi Schur-Pfaffian together with Lemma 4.2 .

Proposition 5.1 Suppose $l \geq 0$ and $r>k+l$. Then $\operatorname{Pf}\left[\vartheta_{r_{1}}^{\left(l_{1}\right)} \cdots \vartheta_{r}^{(l)} \vartheta_{r}^{(l)} \cdots \vartheta_{r_{m}}^{\left(l_{m}\right)}\right]=0$.

Proposition 5.2 (a) Let $i \geq 0$. If $l_{p} \neq \pm i$ for all $p \in\{1, \ldots, m\}$, then $\delta_{i} \operatorname{Pf}\left[\vartheta_{r_{1}}^{\left(l_{1}\right)} \cdots \vartheta_{r_{m}}^{\left(l_{m}\right)}\right]=0$.

(b) Let $i \geq 0$. Suppose that $l_{p} \in\{ \pm i\}$ for some $p \in\{1, \ldots, m\}$ and that $l_{q} \notin\{ \pm i\}$ for all $q \neq p$.

$$
\delta_{i} \operatorname{Pf}\left[\vartheta_{r_{1}}^{\left(l_{1}\right)} \cdots \vartheta_{r_{p}}^{\left(l_{p}\right)} \cdots \vartheta_{r_{m}}^{\left(l_{m}\right)}\right]=\operatorname{Pf}\left[\vartheta_{r_{1}}^{\left(l_{1}\right)} \cdots \vartheta_{r_{p}-1}^{\left(l_{p}-1\right)} \cdots \vartheta_{r_{m}}^{\left(l_{m}\right)}\right]
$$

(c) Let $i>0$. Suppose that $l_{p}=i$ and $l_{q}=-i$ for some $p<q$ and that $l_{s} \neq \pm i$ for all $s \notin\{p, q\}$. Then we have

$$
\begin{aligned}
& \delta_{i} \operatorname{Pf}\left[\vartheta_{r_{1}}^{\left(l_{1}\right)} \cdots \vartheta_{r_{p}}^{(i)} \cdots \vartheta_{r_{q}}^{(-i)} \cdots \vartheta_{r_{m}}^{\left(l_{m}\right)}\right] \\
= & \operatorname{Pf}\left[\vartheta_{r_{1}}^{\left(l_{1}\right)} \cdots \vartheta_{r_{p}-1}^{(i-1)} \cdots \vartheta_{r_{q}}^{(-i-1)} \cdots \vartheta_{r_{m}}^{\left(l_{m}\right)}\right]+\operatorname{Pf}\left[\vartheta_{r_{1}}^{\left(l_{1}\right)} \cdots \vartheta_{r_{p}}^{(i-1)} \cdots \vartheta_{r_{q}-1}^{(-i-1)} \cdots \vartheta_{r_{m}}^{\left(l_{m}\right)}\right] .
\end{aligned}
$$

\subsection{Key lemma}

For $w \in W_{n}^{(k)}$, we denote the corresponding function of the right hand side of 4.2 by $\Theta_{w}^{(n, k)}$. The group $W_{n}^{(k)}$ has a unique longest element denoted by $w_{\max }^{(n, k)}$, which is known to be of order two. Then we can define an involution $W_{n}^{(k)} \rightarrow W_{n}^{(k)}\left(w \mapsto w^{\vee}\right)$ by $w^{\vee}=w w_{\max }^{(n, k)}$. For $w \in W_{\infty}$, let $\delta_{w}:=\delta_{s_{i_{1}}} \cdots \delta_{s_{i_{\ell}}}$ where $w=s_{i_{1}} \cdots s_{i_{\ell}}$ is a reduced word expression.

Lemma 5.3 Let $w \in W_{n}^{(k)}$ and $w_{\max }^{(n, k)}$ the longest element in $W_{n}^{(k)}$. We have $\Theta_{w}^{(n, k)}=\delta_{w^{\vee}} \Theta_{w_{\max }^{(n, k)}}^{(n, k)}$.

Proof: Let $\lambda \in \mathcal{P}_{n}^{(k)}$ be the $k$-strict partition corresponding to $w \in W_{n}^{(k)}$. We proceed by the induction on $\ell\left(w^{\vee}\right)$. The claim obviously holds if $\ell\left(w^{\vee}\right)=0$. Suppose $\ell\left(w^{\vee}\right) \geq 1$. Then there is $i \in\{0,1, \ldots, n-1\}$ such that $w=s_{i} w_{\lambda^{\prime}} \in W_{n}^{(k)}$ for some $\lambda^{\prime} \in \mathcal{P}_{n}^{(k)}$ satisfying $\ell\left(\lambda^{\prime}\right)=\ell(\lambda)+1$. By the induction hypothesis we have

$$
\delta_{w_{\lambda^{\prime}}^{\vee}} \Theta_{\substack{w_{m a x} \\(n, k)}}^{(n, k)}=\sum_{I \subset D\left(\lambda^{\prime}\right)} \operatorname{Pf}\left[\vartheta_{\lambda_{1}^{\prime}+a_{1}^{I}}^{\left(\chi_{1}^{\prime}\right)} \cdots \vartheta_{\lambda_{n-k}^{\prime}+a_{n-k}^{I}}^{\left(\chi_{n-k}^{\prime}\right)}\right]
$$

where $\chi^{\prime}=\left(\chi_{1}^{\prime}, \ldots, \chi_{n-k}^{\prime}\right)$ is the characteristic index of $\lambda^{\prime}$. Lemma 2.2 gives the 4 possible cases of the characteristic index $\chi^{\prime}$ in terms of $\chi$. In each case, we apply $\delta_{i}$ to the right hand side of $(5.1)$ by using Proposition 5.2 and obtain $\Theta_{w}^{(n, k)}$. Since $\delta_{w^{\vee}}=\delta_{i} \delta_{w_{\lambda^{\prime}}}$, the desired formula holds. 


\subsection{Proof of Main Theorem}

First note that the polynomial defined by $\Theta_{w}^{(n, k)}$ is independent of $n$, i.e., for all $m \geq n$, and $w \in W_{n}^{(k)} \subset$ $W_{m}^{(k)}$, we have $\Theta_{w}^{(m, k)}=\Theta_{w}^{(n, k)}$. We can see this fact from the definition of the multi-Schur Pfaffian. Thus, for each $w \in W_{\infty}^{(k)}$, we can define $\Theta_{w}$ to be $\Theta_{w}^{(n, k)}$ by choosing an $n$ so such that $w \in W_{n}^{(k)}$. Proof of the main theorem is completed if we prove the following.

Proposition 5.4 Let $w \in W_{\infty}^{(k)}$. We have $\Theta_{w}=\mathfrak{C}_{w}$.

Proof: By Proposition 2.3. it suffices to show that the family of functions $\Theta_{w}\left(w \in W_{\infty}^{(k)}\right)$ satisfies 2.5$)$ and (2.6).

By observing that $\ell\left(s_{i} w\right)=\ell(w)-1$ implies $\delta_{i} \delta_{w^{\vee}}=\delta_{\left(s_{i} w\right)^{\vee}}$, the first half of the condition 2.5) follows from Lemma 5.3 For the second half of the condition 2.5 , first we see that $\delta_{j} \Theta_{w_{\text {max }}^{(n, k)}}=0$ for all $j \neq k$ from Proposition 5.1 and Proposition 5.2 (a). Now the claim follows from

- If $\ell\left(s_{i} w\right)=\ell(w)+1$ and $s_{i} w \in W_{\infty}^{(k)}$, then $\delta_{i} \delta_{w^{\vee}}=0$.

- If $\ell\left(s_{i} w\right)=\ell(w)+1$ and $s_{i} w \notin W_{\infty}^{(k)}$, then there exists $j \neq k$ such that $\delta_{i} \delta_{w^{\vee}}=\delta_{w^{\vee}} \delta_{j}$.

It remains to show that the condition 2.6 holds. It is obvious that $\left.\Theta_{e}\right|_{e}=1$. For $w \neq 0$, we expand $\Theta_{w}$ as a polynomial in terms of the $\vartheta$-functions $\vartheta_{r}^{(l)}$ by using the definition of Pfaffian. Note that each monomial contains $\vartheta_{\lambda_{1}+a_{1}^{I}+j}^{\left(\chi_{1}\right)}$ as its first factor. We finish the proof by showing the substitution makes this factor vanish by using the following easy observation: $\left.\vartheta_{m}^{(l)}\right|_{\emptyset}=0$ if $\max \{l+k, 0\}<m$.

\subsection{An alternative proof of Main Theorem}

Let $w_{n, 0}$ be the longest element in $W_{n}$. In [IMN11, Theorem 1.2], the authors proved that $\mathfrak{C}_{w_{n, 0}}$ can be obtained from the (factorial) $Q$-function by a certain specialization of variables. In terms of Pfaffian and the theta polynomials ${ }_{k} \vartheta_{r}^{l}:={ }_{k} \vartheta_{r}^{l}(x, z \mid t)$, we have

$$
\mathfrak{C}_{w_{n, 0}}(x, z \mid t)=\operatorname{Pf}\left[{ }_{n-1} \vartheta_{2 n-1 n-2}^{(n-1)} \vartheta_{2 n-3}^{(n-2)} \cdots_{1} \vartheta_{3}^{(1)}{ }_{0} \vartheta_{1}^{(0)}\right] .
$$

Proposition 5.2 and Proposition 5.1 are valid even when the values of $k$ of $\vartheta$ 's are distinct in the Pfaffian. It turns out that the following extra relation of ${ }_{k} \vartheta_{r}^{l}$ allows us to derive the Pfaffian sum formula of $\mathfrak{C}_{w_{m a x}^{(n, k)}}$ by consecutively applying divided difference operators to $\mathfrak{C}_{w_{n, 0}}$ : if $l \geq 0$ and $k>0$,

$$
{ }_{k} \vartheta_{r}^{(l)}={ }_{k-1} \vartheta_{r}^{(l+1)}+\left(t_{l+1}+z_{k}\right) \cdot{ }_{k-1} \vartheta_{r-1}^{(l)} .
$$

Thus Lemma 5.3 implies the main theorem by Theorem 2.1. See [IM15, Section 8]) for the detail.

\section{Acknowledgements}

We would like to thank Dave Anderson, Anders Skovsted Buch, Andrew Kresch, Changzheng Li, Leonardo Mihalcea, and Masaki Nakagawa, Hiroshi Naruse, Harry Tamvakis for helpful conversations and for their comments. 


\section{References}

[AF] D. Anderson and W. Fulton. Degeneracy Loci, Pfaffians, and Vexillary Signed Permutations in Types B, C, and D. arXiv:1210.2066.

[BKTa] Anders Skovsted Buch, Andrew Kresch, and Harry Tamvakis. A Giambelli formula for even orthogonal Grassmannians. arXiv:1109.6669, to appear in J. reine angew. Math.

[BKTb] Anders Skovsted Buch, Andrew Kresch, and Harry Tamvakis. A Giambelli formula for isotropic Grassmanians. arXiv:0811.2781.

[FP98] William Fulton and Piotr Pragacz. Schubert varieties and degeneracy loci, volume 1689 of Lecture Notes in Mathematics. Springer-Verlag, Berlin, 1998. Appendix J by the authors in collaboration with I. Ciocan-Fontanine.

[Ike07] Takeshi Ikeda. Schubert classes in the equivariant cohomology of the Lagrangian Grassmannian. Adv. Math., 215(1):1-23, 2007.

[IM15] T. Ikeda and T. Matsumura. Pfaffian sum formula for symplectic Grassmannians. Math. Z., (doi: 10.1007/s00209-015-1423-x), 2015.

[IMN11] Takeshi Ikeda, Leonardo C. Mihalcea, and Hiroshi Naruse. Double Schubert polynomials for the classical groups. Adv. Math., 226(1):840-886, 2011.

[IN09] Takeshi Ikeda and Hiroshi Naruse. Excited Young diagrams and equivariant Schubert calculus. Trans. Amer. Math. Soc., 361(10):5193-5221, 2009.

[Kaz00] M. Kazarian. On lagrange and symmetric degeneracy loci. Isaac Newton Institute for Mathematical Sciences Preprint Series, 2000.

[Nar] H. Naruse. Private communication.

[Pra91] Piotr Pragacz. Algebro-geometric applications of Schur $S$ - and $Q$-polynomials. In Topics in invariant theory (Paris, 1989/1990), volume 1478 of Lecture Notes in Math., pages 130-191. Springer, Berlin, 1991.

[Tam] H. Tamvakis. Giambelli and degeneracy locus formulas for classical $G / P$ spaces. ar $\chi$ iv: 1305.3543.

[Wil10] E. Wilson. Equivariant Giambelli Formulae for Grassmannians. Ph.D. Thesis, University of Maryland, 2010. 